\title{
Quantized Current Blockade and Hydrodynamic Correlations in Biopolymer Translocation through Nanopores: Evidence from Multiscale Simulations
}

\section{Citation}

Bernaschi, Massimo, Simone Melchionna, Sauro Succi, Maria Fyta, and Efthimios Kaxiras. 2008. "Quantized Current Blockade and Hydrodynamic Correlations in Biopolymer Translocation through Nanopores: Evidence from Multiscale Simulations." Nano Letters 8 (4): 1115-19. https:// doi.org/10.1021/nl073251f.

\section{Permanent link}

http://nrs.harvard.edu/urn-3:HUL.InstRepos:41384053

\section{Terms of Use}

This article was downloaded from Harvard University's DASH repository, and is made available under the terms and conditions applicable to Open Access Policy Articles, as set forth at http:// nrs.harvard.edu/urn-3:HUL.InstRepos:dash.current.terms-of-use\#OAP

\section{Share Your Story}

The Harvard community has made this article openly available.

Please share how this access benefits you. Submit a story.

\section{Accessibility}




\title{
Quantized current blockade and hydrodynamic correlations in biopolymer translocation through nanopores: evidence from multiscale simulations
}

\author{
Massimo Bernaschi ${ }^{1}$, Simone Melchionna ${ }^{2}$, Sauro Succi $^{1}$, Maria Fyta $^{3}$, and Efthimios Kaxiras ${ }^{3}$ \\ 1 Istituto Applicazioni Calcolo, CNR, \\ Viale del Policlinico 137, 00161, Roma, Italy \\ 2 INFM-SOFT, Department of Physics, \\ Università di Roma La Sapienza, \\ P.le A. Moro 2, 00185 Rome, Italy \\ 3 Department of Physics and School of Engineering and Applied Sciences, \\ Harvard University, Cambridge, MA, USA
}

(Dated: October 31, 2018)

\begin{abstract}
We present a detailed description of biopolymer translocation through a nanopore in the presence of a solvent, using an innovative multi-scale methodology which treats the biopolymer at the microscopic scale as combined with a self-consistent mesoscopic description for the solvent fluid dynamics. We report evidence for quantized current blockade depending on the folding configuration and offer detailed information on the role of hydrodynamic correlations in speeding-up the translocation process.
\end{abstract}

Biopolymer translocation through nanoscale pores holds the promise of efficient and improved sensing for many applications in biotechnology, and possibly ultrafast DNA sequencing [1, 2, 3]. Recent advances in fabrication of solid-state nanopores [4, 5] have spurred detailed experimental studies of the translocation process, with DNA as the prototypical biopolymer of interest [6]. Computer simulations that can account for the complexity of the biomolecule motion as it undergoes translocation, as well as its interaction with the environment (the nanopore and the solvent), are crucial in elucidating current experiments [7, 8] and possibly inspiring new ones. Here, we study the dynamical, statistical and synergistic features of the translocation process of a biopolymer through a nanopore by a multiscale method based on molecular dynamics for the biopolymer motion and mesoscopic lattice Boltzmann dynamics for the solvent. We report evidence for quantized current blockade depending on the folding configuration (single- or multi-file translocation) in good agreement with recent experimental observations [7]. Our simulations show the significance of hydrodynamic correlations in speeding-up the translocation process.

Nanopores are an essential element of cells and membranes, controlling the passage of molecules and regulating many biological processes such as viral infection by phages and inter-bacterial DNA transduction [9]. The last two decades have witnessed the emergence of artificial solid-state nanopores as potential devices for sensing biomolecules through novel means [ 6$]$. One of the most intriguing possibilities is ultra-fast sequencing of DNA by measuring the electronic signal as the biomolecule translocates through a nanopore decorated with electrodes [3]. While this goal still remains elusive, a number of detailed studies on DNA translocation through nanopores have been reported recently [7, 8]. These experiments typically measure the blockade of the ion current through the nanopore during the time it takes the molecule to translocate, which provides statistical information about the biomolecule motion during the process.

Numerical simulation of the translocation process provides a wealth of information complementary to experiments, but is hindered by the very large number of particles involved in the full process: these include all the atoms that constitute the biomolecule, the molecules and ions that constitute the solvent, and the atoms that are part of the solid membrane in the nanopore region. The spatial and temporal extent of the full system on atomic scales is far beyond what can be handled by direct computational methods without introducing major approximations. Some universal features of translocation have been analyzed by means of suitably simplified statistical schemes [10], and non-hydrodynamic coarse-grained or microscopic models [11, 12, 13] or other mesoscopic approaches [14]. Many atomic degrees of freedom, and especially those of the solvent and the membrane wall, are uninteresting from the biological point of view. The problem naturally calls for a multi-scale computational approach that can elucidate the interesting experimental measurements while coarse-graining the less important degrees of freedom.

We have developed a multiscale method for treating the dynamics of biopolymer translocation [15] and performed an extensive set of numerical simulations, combining constrained molecular dynamics (MD) for the polymer motion with a Lattice-Boltzmann (LB) treatment of the solvent hydrodynamics [16]. The biopolymer transits through a nanopore under the effect of a localized electric field applied across the pore, mimicking the experimental setup [8]. The simulations provide direct computational evidence of quantized current blockade and confirm the experimentally surmised multiplefile translocation: the molecule passes through the pore in a multi-stranded fold configuration when the pore is sufficiently wide. The simulations offer detailed information about several experimentally difficult issues, in par- 
ticular the role of hydrodynamic correlations in speedingup the translocation process.

A three-dimensional box of size $N_{x} h \times N_{y} h \times N_{z} h$ lattice units, with $h=\Delta x$ the spacing between lattice points, contains the solvent and the polymer. We take $N_{x}=2 N_{y}, N_{y}=N_{z}$; a separating wall is located in the mid-section of the $x$ direction, $x=h N_{x} / 2$. We use $N_{x}=100$ and $N_{0}=400$, where $N_{0}$ is the total number of beads in the polymer. At the center of the separating wall, a cylindrical hole of length $l_{\text {hole }}=10 \mathrm{~h}$ and diameter $d_{p}$ is opened. Three different pore sizes $\left(d_{p}=5 h, 9 h, 17 h\right)$ have been used in the current simulations. Translocation is induced by a constant electric field acting along the $x$ direction and confined to a cylindrical channel of the same size as the hole, and length $l_{p}=12 h$ along the streamwise $(x)$ direction. All parameters are measured in units of the lattice Boltzmann time step and spacing, $\Delta t$ and $\Delta x$, respectively, which are both set equal to 1 . The MD time step is five times smaller than $\Delta t$. The pulling force associated with the electric field in the experiments is $q_{e} E=0.02$ and the temperature is $k_{B} T / m=10^{-4}$. The monomers interact through a Lennard-Jones 6-12 potential with parameters $\sigma=1.8$, and $\epsilon=\times 10^{-4}$ and the bond length among the beads is set at $b=1.2$. The solvent is set at a density $\rho_{L B}=1$, with a kinematic viscosity $\nu_{L B}=0.1$ and a drag coefficient $\gamma=0.1$.

We chose the separation $d$ between the beads to be equal to the persistence length of double-stranded DNA, that is $50 \mathrm{~nm}$, and define the lattice spacing to be $d / 1.2=40 \mathrm{~nm}$. The hole diameters is $3 \Delta x$. The repulsive interaction between the beads and the wall (with parameter $\sigma_{w}=1.5 \Delta x$ [17]) leaves an effective hole of size equal to $\sim 5 \mathrm{~nm}$. Having set the value of $\Delta x$, we choose the time step so that the kinematic viscosity is expressed as: $\nu_{w}=\nu_{L B} \frac{\Delta x^{2}}{\Delta t}$, with $\nu_{w}$ the viscosity of water $\left(10^{-6} \mathrm{~m}^{2} / \mathrm{s}\right)$ and $\nu_{L B}$ the numerical value of the viscosity in LB units; this procedure gives $\Delta t \sim 160 \mathrm{ps}$, with $\nu_{L B}=0.1$. In order to ensure numerical stability, the relation $\gamma \Delta t<1$ must be satisfied. Having established the value of $\Delta t$, we need to adjust the value of the drag coefficient accordingly, $\gamma<6 \cdot 10^{9} \mathrm{sec}^{-1}$. This is significantly smaller than an estimate of the friction based on Stoke's law for DNA [18], which is equivalent to an underdamped system, or an artificially inflated bead mass. This approach is consistent with the coarse graining of the time evolution in the coupled LB-MD scheme.

We focus on the fast translocation regime, in which the translocation time, $t_{x}$, is much smaller than the Zimm time, which is the typical relaxation time of the polymer towards its native (minimum energy, maximum entropy) configuration. This corresponds to the strongfield condition $q_{e} E b / k T>1$. In this regime, simple onedimensional Brownian models [19], or Fokker-Planck representations, cannot apply because the various monomer units do not have time to de-correlate before completing translocation. The ensemble of simulations is generated by different realizations of the initial polymer configuration, to account for the statistical nature of the process.

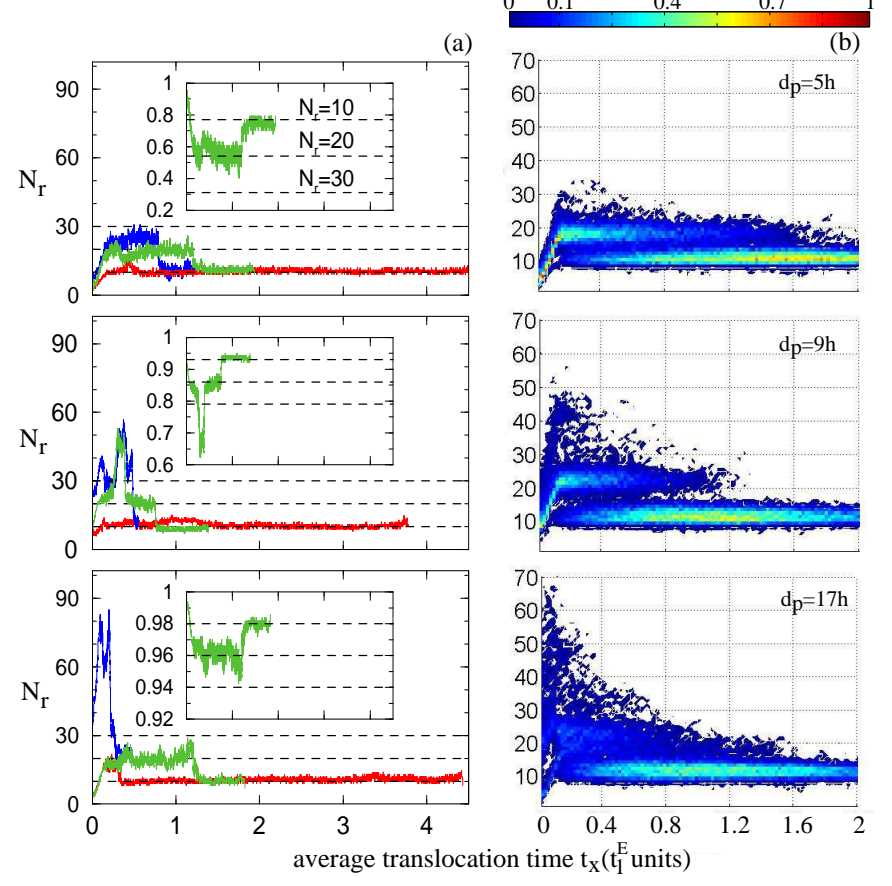

FIG. 1: Number of resident beads with time for three different pore sizes $d_{p}=5 h, 9 h, 17 h$ ( $h=$ lattice spacing) and $N_{0}=400$. (a) The fastest (minimum time, blue), slowest (maximum time, red) and average speed (most probable time, green) translocation events; the insets show the current blockade for the duration of an event with average speed (green) with the current normalized to the open pore value (1). (b) Histogram $P\left(N_{r}, t\right)$ of the distribution of $N_{r}$ with time: short-time trajectories show multi-file character, reaching up to $q \sim 2-8$ in the initial stage of the translocation depending on the pore size; long-time trajectories show little departure from the single-file configuration.

Initially, the polymer is generated by a three-dimensional random walk algorithm with different random numbers for each polymer configuration and one bead chosen randomly constrained at the pore entrance. Then, the polymer is allowed to relax for $\sim 10^{4}$ molecular dynamics steps without including the fluid solvent in the relaxation, while keeping the bead at the pore entrance fixed. We define as time zero $(t=0)$, the time after the relaxation, when the fluid motion is also added, the pulling force begins to act and the translocation process is initiated; at this moment the bead at the pore entrance is also allowed to move. At this stage we do not include any electrostatic interactions within our model for reasons of computational simplicity. As far as the biopolymer motion in the bulk of the solvent is concerned, this may actually be a good approximation of experimental conditions with high salt concentration, which leads to strong screening of electrostatic interactions. The situation at the pore region may require more refined treatment, beyond the scope of the present work.

In Fig. 1(a) we present the number of pore-resident 
beads $N_{r}(t)$ as a function of time, for a narrow $\left(d_{p}=5 h\right)$, mid-sized $\left(d_{p}=9 h\right)$ and large $\left(d_{p}=17 h\right)$ pore, with $h$ the mesh spacing of the lattice Boltzmann simulation, for representative (fastest, slowest and average speed) trajectories. Simulations are repeated over an ensemble of 400 realizations of different initial conditions and for total polymer lengths up to $N_{0}=400$. Time is measured in units of $t_{1}^{E}$, the time it would take for the polymer to translocate if the monomers were to proceed in singlefile configuration at the drift speed; this speed is given by $v_{E}=q_{e} E / \gamma m$, with $q_{e}$ and $m$ the charge and mass of the monomer, $E$ the external electric field and $\gamma$ the hydrodynamic drag. This gives $t_{1}^{E}=b N_{0} / v_{E}=12 N_{0}$ and the number of monomers in the pore for single file translocation is $N_{1}=10$ for the parameters used here.

Fig. 1(a) clearly shows the highly non-linear dynamics of the translocation process: In the initial stage of the translocation, the nanopore gets populated, with the number of resident monomers significantly overshooting the single-file value $N_{1}$, the horizontal dashed lines at heights $q N_{1}$ indicating $q$-file $(q=1,2,3, \ldots)$ translocation. The range of $q$ explored by the translocation trajectories grows approximately with the cross-section of the pore, going from $q \sim 2$ for the smallest pore $d_{p}=5 h$ up to $q \sim 8$ for the largest one $d_{p}=17 h$. Note that these values correspond to about half the maximum allowed q-number, $q_{\max } \sim d_{p} / b$. The fastest events correspond to the largest $q$ value observed, while the slowest events correspond to essentially $q=1$ throughout the translocation. It is also noteworthy that the translocation time typically exceeds the single-file value, $t_{1}^{E}$, except for the fastest events; for the most probable events $q \sim 2$ for all pore sizes indicating that conservative monomermonomer interactions produce an effective slow down compared to a single Langevin particle subject to a constant electric drive and frictional drag $\gamma$.

Fig. 1(a) also presents the current blockade in all three pores for the most probable event in each case, which is the event with a translocation time close to the peak of the distribution over all translocation times. The current blockade is proportional to the number of monomers in the pore per unit area and appears to occur in well defined steps (quantized). Specifically, these blockades are calculated from the difference between the area of the resident beads, $\pi(\sigma / 2)^{2}$, and the total area of the pore, $\pi\left(d_{p} / 2\right)^{2}$. In order to investigate the quantization of the current blockade we monitored the distribution of $N_{r}(t)$ at various time frame intervals of 100 steps. The resident monomers block the current across the channel, so that $N_{r}(t)$ conveys a direct measure of the current drop associated with the biopolymer passage through the nanopore. The corresponding histograms $P\left(N_{r}, t\right)$ for three pore sizes are shown in Fig. 1(b). At early times, these histograms exhibit a multi-peaked structure, which is a clear signature of multi-file translocation. As time passes, the multiple peaks recede in favor of a single-peak distribution, close to the single-file value $N_{1}=10$. This was found to be a stable-attractor for every simulated

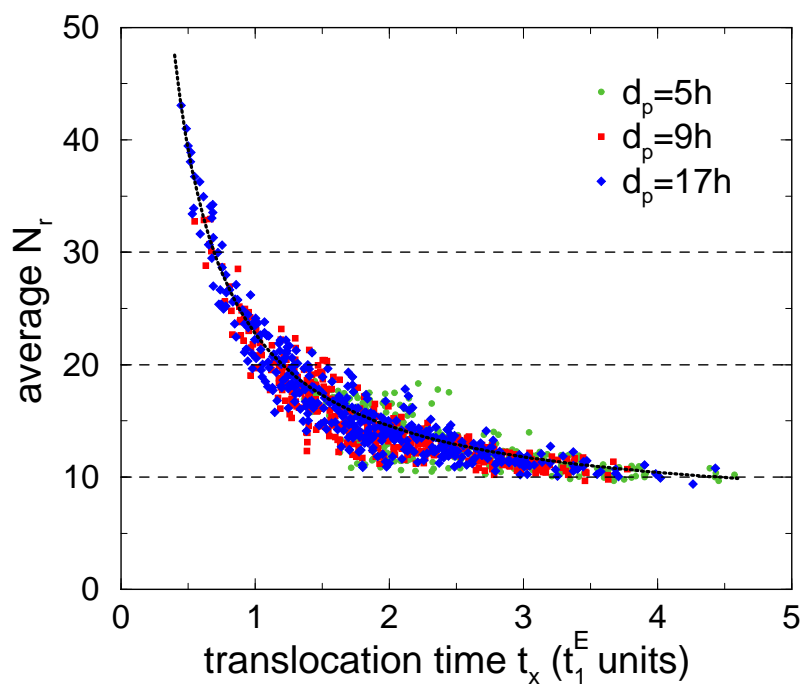

FIG. 2: Scatterplot of the average resident number $N_{r}$ versus translocation time (in units of $t_{1}^{E}$ ) for the ensemble of translocation events for three values of the pore diameter, $d_{p}=5 h, 9 h, 17 h$ and $N_{0}=400$.

trajectory, indicating that the tail of the polymer always translocates as a single-file.

Collecting all results for the average number of resident monomers $\bar{N}_{r}$ as a function of the translocation time $t_{x}$, for the three pore sizes studied, we find a simple relationship, shown in Fig. 2] Experiments [7] have reported that the average number of resident atoms $\bar{N}_{r}$ in each translocation event varies approximately inversely with the duration of translocation $t_{x}$ :

$$
\int_{0}^{t_{x}} N_{r}(t) d t \equiv \bar{N}_{r} t_{x} \propto N_{0}=\text { const. }
$$

The single-file asymptote $N_{1}=10(q=1)$ at longtimes, $t \gg t_{1}^{E}$, is evident. The short-time asymptote, reaching up to $4<q<5$, corresponds to ultrafast translocations $\left(t<t_{1}^{E}\right)$ occurring in the case of the large-diameter pore, $d_{p}=17 h$. These results are intuitively reasonable, since large resident numbers imply that more monomers cross the pore per unit time, hence the translocation becomes faster. The results also support the notion of $N_{r}(t)$ as a measure of the timerate of the translocation, $d N_{T} / d t \propto N_{r}$, from which the inverse-proportionality between $\bar{N}_{r}$ and $t_{x}$ is a direct consequence of $\int_{0}^{t_{x}}\left[d N_{T} / d t\right] d t=N_{0}=$ const. In this expression, $N_{T}(t)$ is the number of translocated monomers at time $t$.

The simulations reveal that solvent correlated motion makes a substantial contribution to the translocation energetics. The role of hydrodynamic correlations is best highlighted by computing the work done by the moving fluid on the polymer (we call this the synergy, $W_{H}$ ) over the entire translocation process as compared to the case 


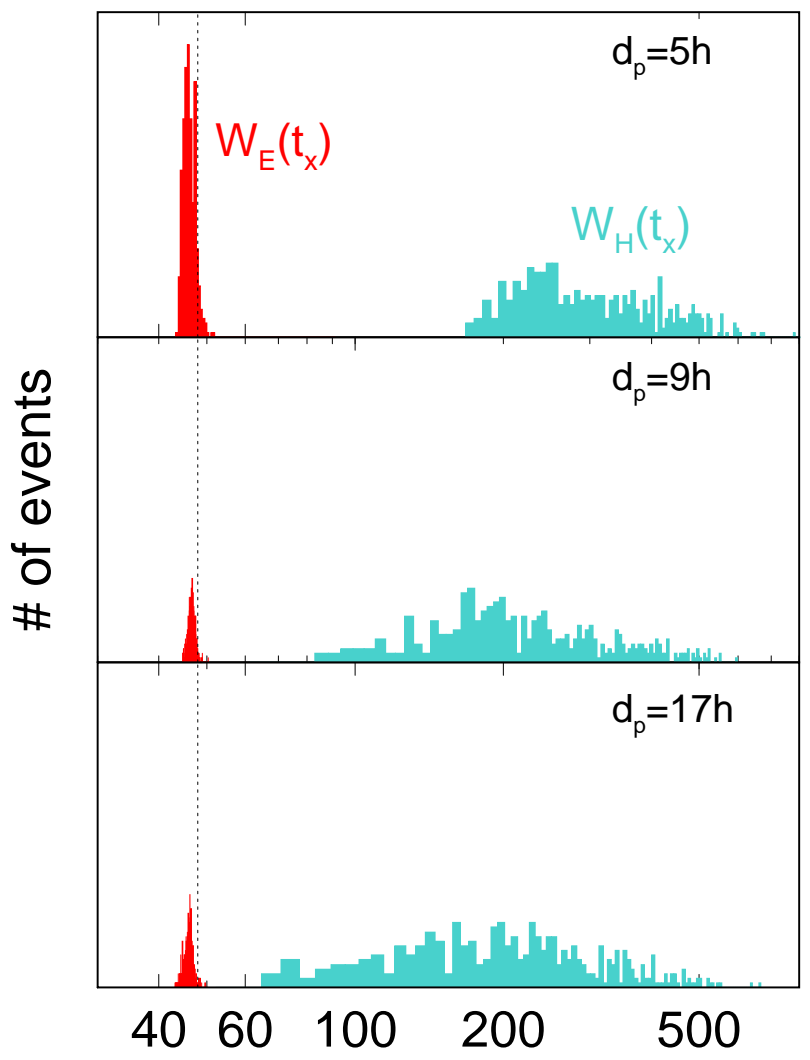

FIG. 3: Statistical distribution of the work performed by the hydrodynamic and electric field during translocation events. The vertical dotted line corresponds to the work $W_{1}^{E}$ done by the electric field on polymers that translocate single-file.

of a passive fluid at rest:

$$
W_{H}\left(t_{x}\right)=\gamma \int_{0}^{t_{x}} \sum_{i=1}^{N_{0}} \vec{u}_{i}(t) \cdot \vec{v}_{i}(t) d t
$$

where $\vec{v}_{i}$ is the velocity of monomer $i$ and $\vec{u}_{i}$ is the fluid velocity at the position of monomer $i$. For the sake of comparison, it is also instructive to contrast $W_{H}$ with the corresponding work done by the electric field

$$
W_{E}\left(t_{x}\right)=q_{e} \int_{0}^{t_{x}} \sum_{i=1}^{N_{r}(t)} \vec{E} \cdot \vec{v}_{i}(t) d t
$$

where the sum extends over the resident monomers only, since the electric field is applied at the pore region only. These statistically averaged values of $W_{H}$ and $W_{E}$ reveal a number of interesting features (see Fig. 3). First, $W_{H}$ is always positive, clearly showing that hydrodynamic correlations provide a cooperative background, as compared to the case of a passive "ether" medium $(\vec{u}=0)$. Second, we observe that the $W_{E}$ has a much narrower distribution of values than $W_{H}$, reflecting the ordered structure of the biopolymer as it passes through the nanopore, as compared to its off-pore morphology. It is useful to introduce
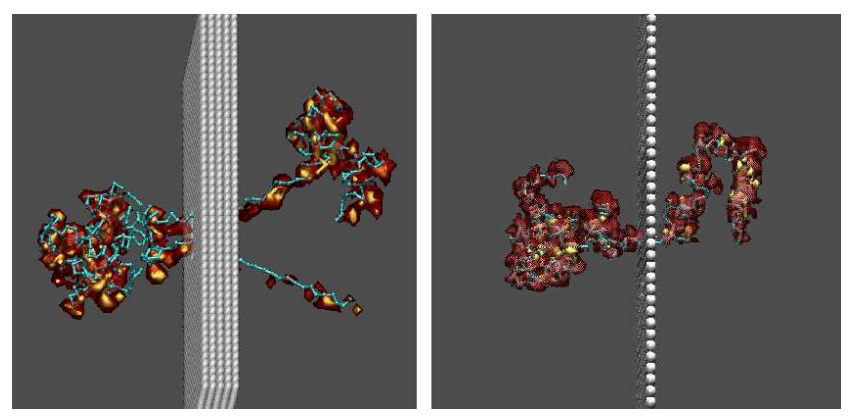

FIG. 4: Left panel: a typical two-folded polymer configuration $\left(d_{p}=9 h\right)$, at time where $65 \%$ of the $N_{0}=400$ total beads have already translocated from right to left; colored contours show the magnitude of the corresponding hydrodynamic synergy field (only five of the nine wall-layers are shown). Right panel: a single-file translocation event for a narrow and shallow pore $\left(d_{p}=3 h, l_{p}=1 h\right)$ with $60 \%$ of the beads translocated and the corresponding magnitude of the synergy.

the work done by the electric field on molecules which translocate single-file and proceed through the pore at speed $v_{E}, W_{1}^{E}=q_{e} E N_{1} v_{E} t_{1}^{E}=b q_{e} E N_{1} N_{0}$. In the absence of any other interaction, a $q$-file translocation at speed $v_{E}$ would complete in a time $t_{x}(q)=t_{1}^{E} / q$ under an electric work $q W_{1}^{E}$. In the present simulations, $W_{1}^{E}=0.12 N_{0}$, thereby $W_{1}^{E}=48$ for $N_{0}=400$. Interestingly, the distribution of $W_{E}$ values is highly peaked at a value very close to $W_{1}^{E}$. The observation that $W^{E} \sim W_{1}^{E}$ implies that $q v_{x}(q) t_{x}(q) \simeq v_{E} t_{1}^{E}$ and since the simulations show that $t_{x}(q)>t_{1}^{E}$, the conclusion is that $v_{x}(q)<v_{E} / q$, indicating that collective motion of the monomers slows down the process.

A major asset of numerical simulations for the study of translocation processes is the direct access to visualization of the morphology of the translocating chain. As an example, we show in Fig. 4 a typical "snapshot" at a time when about $65 \%$ of the monomers have already passed through the pore of a translocating 2 -folded chain of $N_{0}=400$ beads. In the same figure we show for comparison an event for the same length, but for singlefile translocation (unfolded chain) through a very narrow $\left(d_{p}=3 h\right)$ and shallow $\left(l_{p}=1\right)$ pore. In addition to the polymer conformation, we show isocontours of the magnitude of the hydrodynamic synergy density

$$
w_{H}(\vec{r} ; t)=\gamma \sum_{i \in B(\vec{r})} \vec{u}_{i}(t) \cdot \vec{v}_{i}(t)
$$

which is a local (in both space and time) version of the total synergy $W_{H}$ defined in Eq.(2), with $B(\vec{r})$ a grid cell centered around location $\vec{r}=(x, y, z)$. The contours of $w_{H}(\vec{r})$ illustrate the cooperative nature of the hydrodynamic field, with regions of high co-moving flow surrounding the translocating polymer and assisting its motion. This is suggestive of the notion of an "effective" polymer, dressed with the hydrodynamic synergy field, which acts as a self-consistent lubricant, helping 


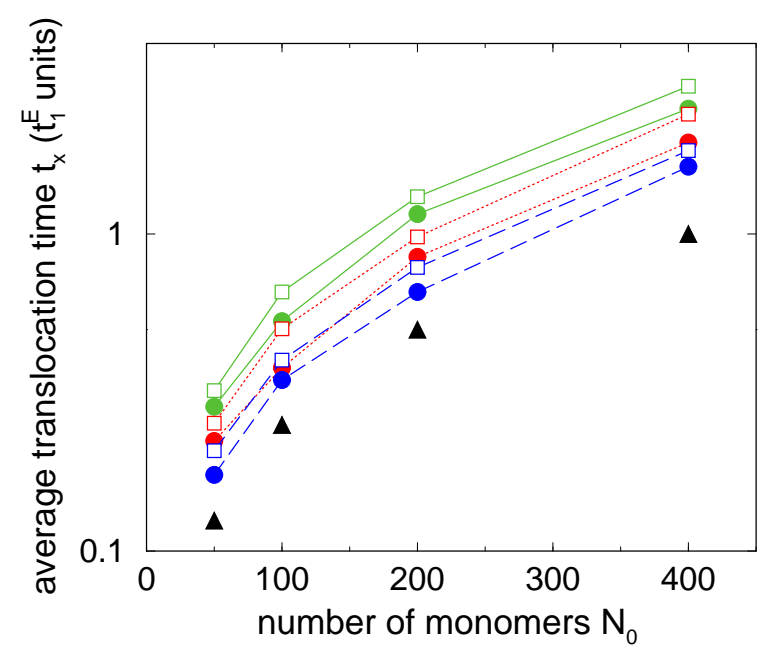

FIG. 5: Average translocation time as a function of polymer length, with (closed circles) and without (open squares) hydrodynamic interactions. Colors correspond to different pore diameters $d_{p}=5 h$ (green solid line), $9 h$ (red dotted line), and $17 h$ (blue dashed line). Black triangles indicate the value of the single-file translocation time $t_{1}^{E}$ for each value of $N_{0}$ with hydrodynamics (all numbers are scaled to the value of $t_{1}^{E}$ for $\left.N_{0}=400\right)$.

the polymer to negotiate a faster passage through the nanopore.

We further investigate this issue by inspecting the average (over an ensemble of 400 realizations) translocation time, $\left\langle t_{x}\right\rangle$, as a function of the polymer length, with and without hydrodynamics. The results are shown in Fig. 5 hydrodynamics consistently accelerates the translocation by roughly $30 \%$ percent. More intuitively, hydrodynamics literally re-normalizes the diameter of the pore: as is clearly visible in Fig. 5, a pore of diameter $d_{p}=5 h$ for a bare polymer (without the hydrodynamic field) is essentially equivalent to a pore of almost double diameter $d_{p}=9 h$ for the hydrodynamically-dressed polymer. In order to assess the degree of correlation between the translocation dynamics of the "dressed" polymer versus the actual one, we have measured the translocated specific synergy (synergy per monomer), defined as

$$
\left\langle w_{H}(t)\right\rangle=\frac{\gamma}{N_{T}(t)} \sum_{i=1}^{N_{T}(t)} \vec{v}_{i}(t) \cdot \vec{u}_{i}(t)
$$

with $N_{T}(t)$ the number of translocated monomers at time $t$. Clearly, any implicit time-dependent functional dependence of the form $\left\langle w_{H}(t)\right\rangle=\left\langle w_{H}\left(N_{T}(t)\right)\right\rangle$ would indicate that mass and synergy translocate in a synchronized manner. We find that the ratio $\langle\vec{u} \cdot \vec{v}\rangle / k T \sim 5$, reflecting the fact that the solvent locally "follows" the monomer and providing a measure of the relative importance of synergistic versus thermal forces. Our results show that $\left\langle w_{H}(t)\right\rangle$ is essentially constant throughout the translocation process. This implies a direct proportionality between the translocated synergy and the number of translocated beads and supports the notion that the "dressed" and the actual polymer proceed in full synchronization across the nanopore.

In conclusion, by using a new multiscale methodology based on the direct coupling of constrained molecular dynamics for the solute biopolymers with a lattice Boltzmann treatment of solvent dynamics, we have been able to confirm a number of experimental observations, such as a direct relation between quantized current blockades and multi-folded polymer conformations during the translocation process. In particular, the simulations reveal an intimate connection between polymer and hydrodynamic motion which promotes a cooperative background for the translocating molecule, thus resulting in a significant acceleration of the translocation process. Such an acceleration can also be interpreted as the outcome of a renormalization of the actual polymer geometry into an effective one, more conducive to translocation. This opens up exciting prospects for the development of optimized nano-hydrodynamic devices based on the finetuning of hydrodynamic correlations. As an example, one may envisage multi-translocation chips, whereby multiple molecules would translocate in parallel across membranes with an array of pores. The optimization of such devices will require control of solvent-mediated moleculemolecule interactions to minimize destructive interference between translocation events.

\section{Acknowledgements}

MB, SM and SS acknowledge support from the Initiative for Innovative Computing and thank the Physics Department of Harvard University for its hospitality. MF acknowledges support by the Nanoscale Science and Engineering Center of the National Science Foundation under NSF Award Number PHY-0117795.
[1] Kasianowicz, J.J. Brandin, E. Branton, D. \& Deamer, D.W. Proc. Natl. Acad. Sci. USA (1996), 93, 13770.

[2] Meller, A. Nivon, L. Brandin, E. Golovchenko, J. \& Branton, D. Proc. Natl. Acad. Sci. USA 2000, 97, 1079.

[3] Deamer, D. W. \& Akeson, M. Trends Biotechnol. 2000, $18,180$.
[4] Li, J. et al. Nature 2001, 412, 166.

[5] Storm, A.J. et al. Nature Mater. 2003, 2, 537.

[6] For a recent review see Dekker, C Nature Nanotechnology 2007, 2, 209.

[7] Li J. et al. Nature Mater. 2003, 2, 611).

[8] Storm, A.J. et al. Nano Lett. 2005, 5, 1734. 
[9] Lodish, H. Baltimore, D. Berk, A. Zipursky, S. Matsudaira, P. \& Darnell, J. Molecular Cell Biology, (W.H. Freeman and Company, New York, 1996).

[10] Sung, W. \& Park, P. J. Phys. Rev. Lett. 1996, 77, 783.

[11] Forrey, C. \& Muthukumar, M. J. Chem. Phys. 2007, 127, 015102.

[12] Matysiak, S. Montesi, A. Pasquali, M. Kolomeisky, A. B. \& Clementi, C. Phys. Rev. Lett. 2006, 96, 118103.

[13] Lubensky, D. K. \& Nelson, D. R. Biophys. J. 1999, 77, 1824.

[14] Reboux, S. Capuani, F. Gonzalez-Segredo, N. Frenkel,
D. J. Chem. Theory Comput. 2006, 2, 495.

[15] Fyta, M.G. Melchionna, S. Kaxiras, E. \& Succi, S. Multiscale Model. and Simul. 2006, 5, 1156.

[16] Benzi, R. Succi, S. \& Vergassola, M. Phys. Rep. 1992, $222,145$.

[17] Weeks J. D., Chandler, D. and Andersen, H. C. J. Appl. Phys., 54, 5237 (1971).

[18] Y. Lu, B. Weers, and N.C. Stellwagen, Biopolymers., 61, 261 (2002).

[19] Kantor, Y. \& Kardar, M. Phys. Rev. E 2004, 69, 021806. 